\title{
Estructura Factorial de la Escala de Satisfacción con la Vida de Diener en Universitarios Mexicanos
}

\author{
Perla J. Jurado, Ma. C. Soto, Miguel Conchas y Raúl J. Nájera* \\ Universidad Autónoma de Chihuahua. Facultad de Ciencias de la Cultura Física. DES Salud CA 101 y 121. \\ ÇCalle Escorza 900, CP 31000 Chihuahua, Chih.- México. (e-mail: pjurado@uach.mx, masoto@uach.mx, \\ mconchas@uach.mx; jnajera@uach.mx) \\ ${ }^{*}$ Autor a quien debe ser dirigida la correspondencia.
}

Recibido Dic. 17, 2018; Aceptado Feb. 26, 2019; Versión final Abr. 10, 2019, Publicado Dic. 2019

\section{Resumen}

El objetivo de esta investigación fue analizar las propiedades psicométricas propuestas por Atienza y colaboradores para la Escala de Satisfacción con la Vida de Diener. La muestra total fue de 400 universitarios mexicanos, con una edad media de 20.54 años ( $D E=1.79$ ). La estructura factorial del cuestionario se analizó a través de análisis factoriales confirmatorios. Los análisis, muestran que una estructura unifactorial es viable y adecuada. La estructura unifactorial, atendiendo a criterios estadísticos y sustantivos, ha mostrado adecuados indicadores de ajuste de fiabilidad y validez. Se concluye que la Escala de Satisfacción con la Vida de Diener puede ser una herramienta útil para avanzar en el estudio de los factores que afectan al bienestar subjetivo.

\section{Factor Structure of the Satisfaction with the Life Scale of Diener in Mexican University Students}

\begin{abstract}
The aim of this research was to analyze the psychometric properties proposed by Atienza and coworkers for the Satisfaction with Life Scale of Diener. The total sample comprised 400 Mexican undergraduate students, with a mean age of 20.54 years $(S D=1.79)$. The factorial structure of the questionnaire has been analyzed through confirmatory factor analyses. These analyses show that an unifactorial structure is viable and adequate. The unifactorial structure, according to statistical and substantive criteria, has shown adequate indicators of reliability and validity adjustment. It is concluded that the Satisfaction with Life Scale of Diener can be a useful tool to advance in the study of the factors that affect subjective well-being.
\end{abstract}




\section{INTRODUCCIÓN}

Por muchos años se ha examinado la pregunta ¿qué es la buena vida? Enfocándose en criterios que definen la calidad de vida tales como amar a los demás, el placer o la autoestima. Otra idea de lo que constituye una buena vida, sin embargo, es que las personas desean pensar que están viviendo buenas vidas. En ese sentido esta definición subjetiva de la calidad de vida es democrática, ya que otorga a cada individuo el derecho de decidir si su vida vale la pena o no. Es pues, desde este enfoque que se define la buena vida y que también ha llegado a llamarse bienestar subjetivo, en términos coloquiales a veces se le denomina felicidad (Diener y Chan, 2011); dicha felicidad incluye algunos componentes como el estar satisfecho con lo que se posee y la manera en que se vive, que le permiten a la persona dar significado y símbolo a su satisfacción con la vida (Martell et. al, 2018). Para Vitterso (2016) la felicidad se diferencia en dos tipos: el tipo uno que incluye estar alegre en su vida, con sentimientos agradables como la bondad y ser feliz con su vida, de acuerdo a la bondad de las propias evaluaciones de la persona; el tipo de felicidad dos hace referencia a tener una vida feliz, donde la bondad se completa de acuerdo con algunos criterios comúnmente acordados. Además, desde la edad antigua, los filósofos han considerado la felicidad como el bien supremo y la motivación esencial para todas las acciones humanas (Anić y Tončić, 2013); pero ¿qué es lo que hace feliz a la gente?, ¿cómo se puede aumentar el bienestar personal? Es algo que se siguen preguntando los investigadores alrededor del mundo; ya que al final de cuentas la búsqueda de la felicidad y el propósito de la vida determinan los pensamientos y las acciones de cada persona (Klug y Mainer, 2014).

Conviene subrayar que la investigación actual sobre el bienestar como tal, se ha derivado en dos enfoques; el primero, refleja la opinión de que el bienestar subjetivo consta de placer, felicidad y la evitación del dolor, el cual se denomina hedonismo y demuestra especial interés en el estudio de los afectos y la satisfacción con la vida, donde el bienestar subjetivo se refiere a las evaluaciones que la gente hace acerca de sus vidas, evaluaciones tanto afectivas como cognitivas; mientras que en el segundo enfoque, el bienestar psicológico no sólo se refiere a la felicidad, sino que éste se encuentra en la actualización de los potenciales humanos, es decir, centra su atención en el desarrollo de las capacidades y el crecimiento personal (Anić y Tončić, 2013; Diener y Chan, 2011; Maddux, 2017; Ryff, 2016). Así pues, existen seis niveles de la persona que son relevantes para que sea óptima, los cuales hacen contribuciones muy importantes al bienestar subjetivo: pertenencia cultural, las relaciones sociales, las autoevaluaciones, los objetivos o metas personales, los rasgos de personalidad y las necesidades psicológicas (Sheldon y Hoon, 2013). Por otro lado, se ha encontrado en muchos casos que la personalidad puede ser asociada más fuertemente con el bienestar subjetivo que con las circunstancias de la vida. En parte, esto podría ser debido al hecho de que el temperamento y otras diferencias individuales pueden influir sobre las sensaciones y las evaluaciones de sus vidas, pero también porque las emociones de la gente son parte inherente de la personalidad (Malkina-Pykh y Pykh, 2013).

La ciencia empírica del bienestar subjetivo, conocida popularmente como felicidad o satisfacción, ha crecido en la última década Recientemente los científicos del comportamiento han centrado su atención no en definir lo que debería ser una buena vida, sino en los factores que llevan a las personas a experimentar subjetivamente sus vidas como valiosas y gratificantes, sin prejuzgar lo que las personas consideran como una buena vida por sí mismas, sino que se basan en los juicios de los propios encuestados proporcionan y que consideran que son importantes (Diener et al., 2018). Existen pues, diferentes factores que afectan la satisfacción con la vida, como el) estrés, la conexión social y el locus de control, etc. (Coccia y Darling, 2014; Dave et al., 2011). De acuerdo con Diener y Chan (2011) las evaluaciones del bienestar subjetivo de las personas incluyen las reacciones emocionales a los eventos, sus estados de ánimo y los juicios que se forman sobre la satisfacción personal, con la vida y en los diferentes dominios tales como el matrimonio y el trabajo. Mientras que Kim-Prieto et al. (2013) afirman que el bienestar subjetivo abarca una amplia gama de componentes, como la felicidad, la satisfacción con la vida, el equilibrio hedónico, la realización y el estrés; el bienestar subjetivo mantiene en su núcleo la evaluación afectiva y cognitiva de la propia vida; además se extiende desde lo específico y concreto hasta lo global y abstracto, es decir, experiencias momentáneas de las personas sobre sus vidas frente a juicios globales. Esto no es sorprendente, ya que las personas pueden estar satisfechas con su vida en lo general y, al mismo tiempo, pueden estar satisfechas e insatisfechas con situaciones particulares de su vida.

En la transición de la educación básica a la educación superior, los estudiantes de primer año experimentan estrés debido al entorno académico, cultural y social al que deben adaptarse, lo que puede afectar negativamente a su bienestar subjetivo, que a su vez puede influir negativamente en el rendimiento académico y aumentar la probabilidad de abandono (De Coninck et al., 2019). Además los estudiantes de medicina y psicología reportan altos niveles de angustia mental y bajos niveles de satisfacción con la vida, y ya que como profesionales de la salud es muy probable que sigan informando altos niveles de estrés, agotamiento y suicidio en relación a la población general; donde las fuentes de estrés incluyen alto número de casos, altas expectativas de rendimiento, presión con el tiempo, fracaso del tratamiento y exposición continua al sufrimiento humano (De Vibe et al., 2018; Peluso et al., 2011). 
Diversos estudios relacionan al bienestar subjetivo con distintas variables, por ejemplo, se encontró que una puntuación alta en bienestar subjetivo promueve la resiliencia (Bajaj y Pande, 2016); a mayor bienestar subjetivo, menor depresión, menor ansiedad y menos síntomas físicos y un mayor alcance de metas (Boudreaux y Ozer, 2013); el bienestar subjetivo promueve la buena salud física y mental (De la Cruz et al., 2011; Salsman et al., 2014); además se ha reportado que el bienestar subjetivo junto con la motivación y la adaptación predicen el logro y el rendimiento académico (Bailey y Phillips, 2016; Portolés y González, 2015); también se ha informado que el locus de control, la ansiedad y la autoestima se correlacionaron significativamente con el bienestar subjetivo (Pu et al., 2017); asimismo se ha reportado que la actividad física contribuye al aumento del bienestar subjetivo (Wicker y Frick, 2015).

En cuanto a instrumentos para la medición del bienestar subjetivo se refiere, existe una gran cantidad de ellos, tales como Global Well-Being Inventory [GWB] (Omar et al., 2009), The Pemberton Happiness Index [PHI] (Paiva et al., 2016), The Positive and Negative Affect Schedule [PANAS] (Crawford y Henry, 2004), en México se han desarrollado instrumentos como: la Escala Multidimensional para la Medición del Bienestar Subjetivo [EMMBSAR] (Anguas, 2001) y el Bienestar autorreportado [BIARE] (Instituto Nacional de Estadística y Geografía, 2012). Sin embargo uno de los instrumentos clásicos y más usados para la medición del bienestar subjetivo es la Escala de Satisfacción con la Vida de Diener. Desde su introducción en 1985, la Escala de Satisfacción con la Vida se ha utilizado considerablemente como medida del componente de satisfacción con la vida del bienestar subjetivo. Se ha demostrado que las puntuaciones en dicha escala se correlacionan con las medidas de salud mental y predicen comportamientos futuros, como los intentos de suicidio. En el área de la psicología de la salud, la Escala de satisfacción con la vida se ha utilizado para examinar la calidad de vida subjetiva de las personas que experimentan serios problemas de salud (Anić y Tončić, 2013; AyyashAbdo y Sánchez, 2012; Casas et al., 2013; Erilmaz, 2012; Gere y Schimmack, 2013; Klug y Mainer, 2014).

El bienestar es pues, un tema importante de investigación en varias disciplinas, lo que refleja el creciente reconocimiento de su gran valor en los principales dominios de la vida. Por lo tanto se considera trascendente explorar la composición factorial de la Escala de Satisfacción con la Vida de Diener en universitarios mexicanos, ya que éstos se enfrentan a presiones en todos los elementos de su entorno; y la manera en que se acercan a su entorno sociofísico puede influir en su satisfacción general con la vida (Coccia y Darling, 2014). Por otro lado, la importancia intercultural de la felicidad y la satisfacción se puede inferir de su aparición en discusiones filosóficas a través de muchas tradiciones culturales, y se ha encontrado que los componentes del bienestar psicológico correlacionan en la mayoría de las culturas, dichas similitudes interculturales son relevantes porque resaltan nuestra humanidad común (Tov y Diener, 2013). Por lo que, comprender los factores de la satisfacción con la vida en jóvenes universitarios cobra una gran relevancia.

Son varios los estudios en los que la Escala de Satisfacción con la Vida de Diener se ha validado en diversas poblaciones y ha mostrado adecuadas propiedades psicométricas, así como una estructura unidimensional, por ejemplo, en Europa Di Fabio y Gori (2016) examinaron las propiedades psicométricas en una muestra de 1515 adolescentes italianos, los resultados confirmaron una buena confiabilidad y validez concurrente. En América Latina Álvarez y colaboradores (2018) realizaron un estudio transversal con 23 adolescentes entre 10 y 18 años, encontrando valores en los indicadores del modelo: $\mathrm{X}^{2} / \mathrm{gl}=1.856, \mathrm{RMR}=.003, \mathrm{GFI}=.98, \mathrm{NFI}=.97$, y el coeficiente de consistencia interna alpha de Cronbach fue de .81. Sin embargo, en el contexto mexicano no se encontró una validación de la escala en estudiantes universitarios, aunque sí se localizó una investigación realizada en estudiantes de preparatoria por Martell y colaboradores (2018), donde los valores en los indicadores del modelo fueron: $\mathrm{X}^{2} / \mathrm{gl}=5.291$, $\mathrm{SRMR}=.029, \mathrm{RMR}=.065$, GFI=.998, $\mathrm{AGFI}=.993$, y el coeficiente de consistencia interna alpha de Cronbach de .85 .

Así pues, el presente estudio clasificado como investigación instrumental (Ato, López y Benavente, 2013) se ha dirigido a proporcionar apoyo empírico a la división factorial de la Escala de Satisfacción con la Vida adaptada por Atienza et al. (2000) esto ante la necesidad de comprobar la estructura factorial de un instrumento y la equivalencia psicométrica del mismo en distintos grupos; ya que en el contexto de la comparación intergrupal, es indispensable plantearse a necesidad de llevar a cabo la adaptación de un instrumento de medida psicológica que cumpla con los criterios de equivalencia, pero sobre todo establecer si la misma estructura factorial es aplicable a distintos grupos de sujetos 0 , en general a distintas poblaciones (Byrne, 2016).

\section{METODOLOGÍA}

A continuación se presenta la metodología utilizada en la investigación en donde se describe a los participantes, el instrumento utilizado, el procedimiento realizado y el análisis de los datos. 


\section{Participantes}

Participaron en el estudio 400 jóvenes, 268 mujeres y 132 hombres, todos estudiantes universitarios de México. Los participantes se seleccionaron mediante un muestreo por conveniencia entre los estudiantes de la Licenciatura en Psicología de la Facultad de Psicología de la Universidad Autónoma de Yucatán; se seleccionó el muestreo por conveniencia con el fin de lograr representar de mejor manera la población a estudiar y por la facilidad de utilizar a los grupos predefinidos por las cohortes generacionales. La edad de los participantes fluctuó entre los 17 y 27 años, con una media de 20.54 y una desviación típica de 1.79 años.

\section{Instrumento}

La Escala de Satisfacción con la Vida (por sus siglas en inglés "Satisfaction With Life Scale" (SWLS)) de Diener et al. (1985), adaptada por Atienza et al. (2000) escala tipo Likert de cinco ítems que evalúa el juicio global que hacen las personas sobre la satisfacción con su propia vida, en su análisis los autores reportaron una muy buena consistencia interna con un alpha de Cronbach de .84, los enunciados de los ítems se muestran en la tabla 1; Para nuestro estudio se hicieron dos adaptaciones a la versión de Atienza et al. (2000): Primera adaptación, en la versión original se puntúa con cinco opciones de respuesta: (1) totalmente en desacuerdo, (2) en desacuerdo, (3) ni de acuerdo ni en desacuerdo, (4) de acuerdo y (5) totalmente de acuerdo; en la versión utilizada en la presente investigación el participante elige entre 11 posibles respuestas. Conjugamos la escala original con nuestra versión para que quedara de la manera siguiente: completamente en desacuerdo (0), en desacuerdo (1,2 y 3), ni de acuerdo ni en desacuerdo (4, 5 y 6$)$, de acuerdo (7, 8 y 9$)$ y completamente de acuerdo (10). Esta primera adaptación se justifica porque las participantes al ser estudiantes están acostumbrados a la escala de 0 a 10, ya que así han sido evaluadas por el sistema educativo de nuestro país (México), figura 1. La segunda adaptación consistió en aplicar el instrumento por medio de una computadora; esto con el fin de permitir el almacenamiento de los datos sin etapas previas de captura, con una mayor precisión y rapidez.

Tabla 1: Ítems de la Escala de Satisfacción con la Vida

1. En la mayoría de los aspectos de mi vida es como yo quiero que sea.

2. Las circunstancias de mi vida son muy buenas.

3. Estoy satisfecho con mi vida.

4. Hasta ahora he conseguido de la vida las cosas que considero importantes.

5. Si pudiera vivir mi vida otra vez, no cambiaría casi nada.

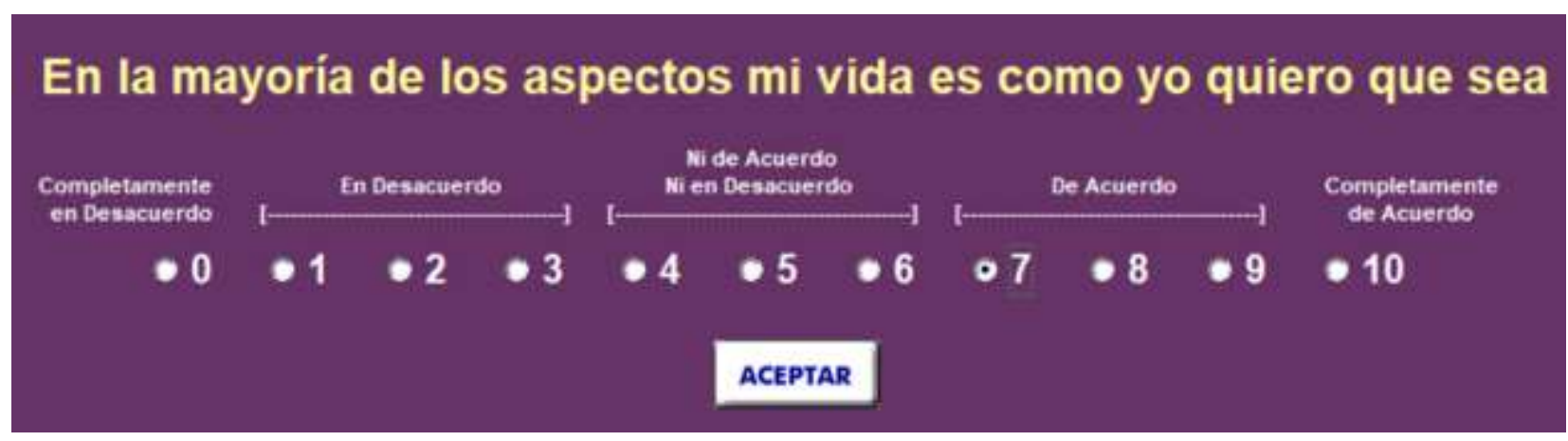

Fig. 1: Ejemplo de respuesta para los ítems del cuestionario.

\section{Procedimiento}

Se invitó a participar en el estudio al alumnado de licenciatura en Psicología de la Facultad de Psicología de la Universidad Autónoma de Yucatán (México). Los que aceptaron participar firmaron la carta de aceptación correspondiente. Luego se aplicó el instrumento, antes descrito, por medio de una computadora personal (módulo administrador del instrumento del editor de escalas de ejecución típica), en una sesión de aproximadamente 10 minutos. Al inicio de la sesión se hizo una pequeña introducción sobre la importancia de la investigación y de cómo acceder al instrumento. Se les solicitó la máxima sinceridad y se les garantizó la confidencialidad de los datos que se obtuvieran. Las instrucciones de cómo responder se encontraban en las primeras pantallas; antes del primer reactivo del instrumento. Al término de la sesión se les agradeció su participación. Una vez aplicado el instrumento se procedió a recopilar los resultados por medio del módulo generador de resultados del editor de escalas versión 2.0 (Blanco et al., 2013). 


\section{Análisis de datos}

El primer paso del análisis de las propiedades psicométricas del cuestionario consistió en calcular la asimetría y la curtosis de cada ítem, para determinar si se cumplía el supuesto de normalidad. Luego, se sometieron a comparación dos modelos de medida: el SWLS-M1, que responde a una estructura unifactorial acorde a la distribución original de los ítems dentro del cuestionario y el SWLS-M1F, que corresponde a la estructura factorial del modelo anterior sin el ítem 4 de más baja saturación en el factor.

Para conducir todos los análisis factoriales confirmatorios se utilizó el software AMOS 21 (Arbuckle, 2012), las varianzas de los términos de error fueron especificados como parámetros libres, en la variable latente (factor) se fijó uno de los coeficientes estructurales asociados a uno, para que su escala sea igual a la de una de las variables observables (ítems). El método de estimación empleado fue el de Máxima Verosimilitud (ML) con la aplicación de procedimientos de remuestreo bootstrap para los casos de no normalidad (Byrne, 2016) aun cuando en AMOS 21.0 el ML es especialmente robusto para posibles casos de no normalidad, especialmente si la muestra es suficientemente amplia y los valores de asimetría y curtosis no son extremos (asimetría < |2| y curtosis < |7|); siguiendo además la recomendación de Byrne (2016), en el sentido de que cuando se emplea análisis factorial confirmatorio se debe corroborar no sólo el ajuste de un modelo teórico sino que es recomendable comparar los índices de ajuste de varios modelos alternativos para seleccionar el mejor.

Para evaluar el ajuste del modelo se emplearon el estadístico Chi-cuadrado, el índice de bondad de ajuste (GFI) y el error cuadrático medio de aproximación (RMSEA) como medidas absolutas de ajuste. El índice de bondad ajustado (AGFI), el Índice Tucker-Lewis (TLI) y el índice de bondad de ajuste comparativo (CFI) como medidas de ajuste incremental. La razón de Chi-cuadrado sobre los grados de libertad (CMIN/GL) y el Criterio de Información de Akaike (AIC) como medidas de ajuste de parsimonia (Byrne, 2016). Los valores considerados como aceptables son: para los índices GFI, AGFI, TLI, CFI .90 se considera un modelo aceptable y .95 un modelo óptimo; para el RMSEA entre .05 y .08 indica un buen ajuste del modelo (Schumacker y Lomax, 2012). Por último se calculó la fiabilidad del factor del mejor de los modelos de medida obtenido, a través del Coeficiente Alpha de Cronbach (Elosua y Zumbo, 2008) y del Coeficiente Omega (Revelle y Zinbarg, 2009).

\section{RESULTADOS}

En esta sección se detallan los hallazgos sobre los análisis descriptivos e índices de discriminación y los análisis factoriales confirmatorios.

\section{Análisis descriptivos e índices de discriminación}

En la Tabla 2 se resumen los valores de asimetría y curtosis de las cinco variables contempladas en el modelo de medida. Todas las variables muestran valores de asimetría de \pm 1.25 y \pm 2.10 de curtosis, por lo que se infiere que las variables se ajustan razonablemente a una distribución normal; por otro lado el índice multivariado de Mardia por debajo del valor 70 indica normalidad multivariada (Rodríguez y Ruiz, 2008).

Tabla 2: Asimetría y curtosis de los ítems del cuestionario SWLS.

Nota: $A S$ = asimetría; $C U$ = curtosis

\begin{tabular}{|l|c|c|}
\hline \multicolumn{1}{|c|}{ Ítem } & AS & CU \\
\hline Ítem 1 & -1.25 & 1.83 \\
\hline Ítem 2 & -0.98 & 0.88 \\
\hline Ítem 3 & -1.33 & 2.08 \\
\hline Ítem 4 & -1.15 & 1.74 \\
\hline Ítem 5 & -0.88 & 0.03 \\
\hline índice multivariado de Mardia & & 17.85 \\
\hline
\end{tabular}

\section{Análisis factoriales confirmatorios}

Los resultados globales del análisis factorial confirmatorio (GFI .986; RMSEA .067; CFI .991) para el modelo SWLS-M1 que corresponde a una estructura unifactorial acorde a la distribución original de los ítems dentro del cuestionario, indican que el modelo de medición es óptimo (Tabla 3). En la Tabla 3, Nota: ${ }^{\star} p<.05$; GFI = índice de bondad de ajuste; RMSEA = error cuadrático medio de aproximación; AGFI = índice corregido de la bondad de ajuste; TLI = índice de Tucker-Lewis; CFI = índice de ajuste comparativo; CMIN/DF = índice de ajuste chi cuadrado dividido por los grados de libertad; AIC = criterio de información de Akaike. 
Tabla 3 Índices absolutos, incrementales y de parsimonia para los modelos generados.

\begin{tabular}{|c|c|c|c|c|c|c|c|c|c|}
\hline & \multicolumn{3}{|c|}{ Índices absolutos } & \multicolumn{3}{c|}{ Índices incrementales } & \multicolumn{3}{c|}{ Índices de parsimonia } \\
\hline Modelo & $\chi^{2}$ & GFI & RMSEA & AGFI & TLI & CFI & & CMIN/DF & AIC \\
\hline SWLS-M1 & $13.872^{*}$ & .986 & .067 & .959 & .982 & .991 & 2.774 & 33.872 \\
\hline SWLS-M1F & 4.154 & .995 & .052 & .975 & .992 & .997 & & 2.077 & 20.154 \\
\hline
\end{tabular}

El factor del modelo SWLS-M1 explica aproximadamente el $66 \%$ de la varianza y de acuerdo a los resultados obtenidos 3 de los 5 ítems saturan por encima de .70 en su dimensión prevista (Tabla 4). Los resultados globales del análisis factorial confirmatorio (GFI .995; RMSEA .052; CFI .997) del segundo modelo sometido a prueba (SWLS-M1F) que corresponde a la estructura factorial del modelo anterior sin el ítem 4 de más baja saturación en el factor, indican que este modelo de medición es mejor que el modelo anterior y que su ajuste es óptimo (Tabla 3). El factor de este modelo explica aproximadamente el $71 \%$ de la varianza. Por otro lado, solo uno de los ítems satura por debajo de .70 en su dimensión prevista (Tabla 4). El factor del modelo SWLSM1, obtiene valores de consistencia interna de $\alpha=.857$ y $\Omega=.874$ y el factor del modelo SWLS-M1F, obtiene valores de consistencia interna de $\alpha=.840$ y $\Omega=.865$.

Tabla 4: Soluciones estandarizadas análisis factorial confirmatorio para los Modelos SWLS-M1 y SWLS-M1F.

\begin{tabular}{|l|c|c|}
\hline & \multicolumn{2}{|c|}{ Pesos Factoriales } \\
\hline \multicolumn{1}{|c|}{ Ítem } & SWLS-M1 & SWLS-M1F \\
\hline 1. En la mayoría de los aspectos mi vida es como yo quiero que sea & .80 & .80 \\
\hline 2. Las circunstancias de mi vida son muy buenas & .74 & .75 \\
\hline 3. Estoy satisfecho con mi vida & .91 & .90 \\
\hline 4. Hasta ahora he conseguido de la vida las cosas que considero importantes & .67 & - \\
\hline 5. Si pudiera vivir mi vida otra vez no cambiaría casi nada & .68 & .68 \\
\hline
\end{tabular}

\section{DISCUSIÓN}

El objetivo principal del estudio fue indagar si se corresponden o no los resultados psicométricos propuestos por Atienza et al. (2000) para la Escala de Satisfacción con la Vida de Diener a través de una muestra de universitarios utilizando el análisis factorial confirmatorio (AFC). Los análisis factoriales confirmatorios realizados apoyan la estructura unifactorial, donde el factor obtenido presenta en general saturaciones factoriales estandarizadas adecuadas, saturaciones que se corresponden con la estructura propuesta para el cuestionario por Atienza et al. (2000); no obstante la necesidad de eliminar el ítem 4 (hasta ahora he conseguido de la vida las cosas que considero importantes) por su baja saturación, ya que la condición óptima se presenta cuando las saturaciones son superiores a .7 (Lloret-Segura et al., 2014). Además, el factor en el modelo de cuatro ítems evidenció una consistencia interna muy aceptable, a pesar del número reducido de ítems. En síntesis, el análisis de las propiedades psicométricas de la Escala de Satisfacción con la Vida de Diener, ha mostrado una validez y confiabilidad aceptable, estudios similares también han reportado la fiabilidad y validez de la escala como el realizado por Di Fabio y Gori (2016) en Europa con una muestra de 1515 adolescentes; Álvarez et al. (2018) en América Latina con 200 sujetos; y en México el realizado por Martell et al. (2018) con 428 estudiantes donde encontraron que las cargas de los ítems van desde .58 a .88, encontrando que el ítem 5 fue el ítem con la carga factorial más baja, al igual que en el estudio realizado por Di Fabio y Gori (2016), a diferencia del estudio de Álvarez et al. (2018) donde el ítem 4 fue el de saturación más baja resultados similares a los encontrados en el presente estudio.

\section{CONCLUSIONES}

En conclusión una estructura de un solo factor es viable y adecuada de acuerdo a los requisitos psicométricos establecidos. La estructura unifactorial, atendiendo a criterios estadísticos y sustantivos, ha mostrado adecuados indicadores de ajuste, de fiabilidad y de validez. Sin embargo, el alcance de estos resultados es limitado, y es necesario que en investigaciones futuras se confirme la estructura obtenida, lo cual permitirá contar con evidencia más robusta respecto a la estructura factorial de la escala. Específicamente, debe demostrarse si la invarianza de la estructura de la escala se cumple por género, edad, entre alumnos de distintas licenciaturas, entre otras; de tal manera que, se considera que más estudios son necesarios con el fin de corroborar o refutar los datos obtenidos en las investigaciones realizadas hasta el momento. Asimismo, es indispensable comprobar si la escala resulta útil para estudiar la relación entre satisfacción con la vida y otras variables psicosociales de importancia. 


\section{AGRADECIMIENTOS}

A la Secretaría de Educación Pública-Subsecretaría de Educación Superior-Dirección de Superación Académica- Programa para el Desarrollo Profesional Docente (DE-13 -6894) quién financió este estudio.

\section{REFERENCIAS}

Álvarez C. A., A. M. Briceño, y otros tres autores, Estudio De Adaptación Y Validación Transcultural De Una Escala De Satisfacción Con La Vida Para Adolescentes, doi: 10.4067/S0370-41062018000100051 Revista chilena de pediatría, 89(1), 51-58, (2018).

Anguas A. M., Identificación Y Validación Del Significado Del Bienestar Subjetivo En México: Fundamentos Para El Desarrollo De Un Instrumento De Medición, Interamerican Journal of Psychology, 35(1), 163-183, (2001).

Anić P. y M. Tončić, Orientations to Happiness, Subjective Well-Being and Life Goals, doi: 159.942.072-057.875, Psihologijske teme, 22(1), 135-153, (2013).

Arbuckle J. R., Amos Users Guide Version 21.0, Marketing Department, SPSS Incorporated, Chicago, IL, (2012).

Atienza F. L., D. Pons, I. Balaguer y M. García-Merita, Propiedades Psicométricas De La Escala De Satisfacción Con La Vida En Adolescentes, Psicothema, 12(2), 314-319, (2000).

Ato M., J. J. López y A. Benavente, Un Sistema De Clasificación De Los Diseños De Investigación En Psicología, Anales de psicología, 29(3), 1038-1059, (2013).

Ayyash-Abdo H. y M.-J. Sánchez-Ruiz, Subjective Wellbeing and Its Relationship with Academic Achievement and Multilinguality among Lebanese University Students, doi: 10.1080/00207594.2011.614616, International Journal of Psychology, 47(3), 192-202, (2012).

Bailey T. H. y L. J. Phillips, The influence of motivation and adaptation on students' subjective well-being, meaning in life and academic performance, doi: 10.1080/07294360.2015.1087474, Higher education research \& development, 35(2), 201216, (2016).

Bajaj B. y N. Pande, Mediating role of resilience in the impact of mindfulness on life satisfaction and affect as indices of subjective well-being, doi: 10.1016/j.paid.2015.09.005, Personality and Individual Differences, 93, 63-67, (2016).

Blanco H., M. Ornelas, J. y otros cinco autores, Editor for Creating and Applying Computerise Surveys, doi: 10.1016/j.sbspro.2013.12.105, Procedia Social and Behavioral Sciences, 106, 935-940, (2013).

Boudreaux M. J. y D. J. Ozer, Goal Conflict, Goal Striving, and Psychological Well-Being, doi: 10.1007/s11031-012-93332, Motivation and Emotion, 37, 433-443, (2013).

Byrne B. M., Structural Equation Modeling with Amos: Basic Concepts, Applications, and Programming, Routledge, New York, NY, (2016).

Casas F., S. Fernández-Artamendi, y otros cuatro autores, El Bienestar Subjetivo En La Adolescencia: Estudio Comparativo De Dos Comunidades Autónomas En España., doi: 10.6018/analesps.29.1.145281, Anales de psicología, 29(1), 148-158, (2013).

Coccia C. y C. A. Darling, Having the Time of Their Life: College Student Stress, Dating and Satisfaction with Life, doi: doi:10.1002/smi.2575, Stress and Health, 32(1), 28-35, (2014).

Crawford J. R. y J. D. Henry, The Positive and Negative Affect Schedule (Panas): Construct Validity, Measurement Properties and Normative Data in a Large Non-Clinical Sample, British Journal of Clinical Psychology, 43(3), 245-265, (2004).

Dave R., K. N. Tripathi, P. Singh y R. Udainiya, Subjective Well-Being, Locus of Control and General Self-Efficacy among University Students, Amity Journal of Applied Psychology, 2, 28-32, (2011).

De Coninck D., K. Matthijs y P. Luyten, Subjective well-being among first-year university students: A two-wave prospective study in Flanders, Belgium, doi: 10.5204/ssj.v10i1.642, Student Success, 10(1), 33-45, (2019).

De la Cruz-Sánchez E., M. I. Moreno-Contreras, J. Pino-Ortega y R. Martínez-Santos, Actividad Física Durante El Tiempo Libre Y Su Relación Con Algunos Indicadores De Salud Mental En España, Salud Mental, 34(1), 45-52, (2011).

De Vibe M., I. Solhaug, y otros cuatro autores, Six-year positive effects of a mindfulness-based intervention on mindfulness, coping and well-being in medical and psychology students; Results from a randomized controlled trial, PloS one, 13(4), e0196053, (2018).

Diener E. y M. Y. Chan, Happy People Live Longer: Subjective Well-Being Contributes to Health and Longevity, doi: 10.1111/j.1758-0854.2010.01045.x, Applied Psychology: Health and Well-Being, 3(1), 1-43, (2011).

Diener E., R. A. Emmons, R. J. Larsen y S. Griffin, The Satisfaction with Life Scale, Journal of Personality Assessment, 49(1), 71-75, (1985)

Diener, E., S. Oishi, y L. Tay, Advances in subjective well-being research, doi: 10.1038/s41562-018-0307-6, Nature Human Behaviour, 2(4), 253-260, (2018). 
Di Fabio A. y A. Gori, Measuring Adolescent Life Satisfaction: Psychometric Properties of the Satisfaction with Life Scale in a Sample of Italian Adolescents and Young Adults, doi: 10.1177/0734282915621223, Journal of Psychoeducational Assessment, 34(5), 501-506, (2016).

Elosua P. y B. D. Zumbo, Coeficientes De Fiabilidad Para Escalas De Respuesta Categórica Ordenadas, Psicothema, 20(4), 896-901, (2008).

Erilmaz A., A Model of Subjective Well-Being for Adolescents in High School, doi: 10.1007/s10902-011-9263-9, Journal of Happiness Studies, 13, 275-289, (2012).

Gere J. y U. Schimmack, When Romantic Partners' Goals Conflict: Efects on Relationship Quality and Subjective WellBeing, doi: 10.1007/s10902-011-9314-2, Journal of Happiness Studies, 14, 37-49, (2013).

Instituto Nacional de Estadística y Geografía. Bienestar Subjetivo. Instituto Nacional de Estadística y Geografía; 2012.

Kim-Prieto C., E. Diener, y otros tres autores, Integrating the Diverse Definitions of Happiness: A Time-Sequential Framework of Subjective Well-Being, En The Exploration of Happiness: Present and Future Perspectives, por A. F. Delle, pp 47-75 Springer, (2013).

Klug H. J. P. y G. W. Mainer, Linking Goal Progress and Subjective Well-Being: A Meta-Analysis, doi: 10.1007/s10902013-9493-0, Journal of Happiness Studies, 16(1), 37-65, (2014).

Lloret-Segura S., A. Ferreres-Traver, A. Hernández-Baeza y I. Tomás-Marco, El análisis factorial exploratorio de los ítems: una guía práctica, revisada y actualizada, Anales de psicología, 30(3), 1151-1169, (2014).

Maddux J. E., Subjective well-being and life satisfaction: An introduction to conceptions, theories, and measures, En Subjective Well-Being and Life Satisfaction, por J. E. Maddux, Taylor \& Francis, (2017).

Malkina-Pykh I. G. y Y. A. Pykh, Integrated Modelling for Delineating Index of Subjective Well-Being: Psychological Predictors and Method of Response Functions, doi: 10.1016/j.ecolind.2012.04.003, Ecological Indicators, 28, 150-158, (2013).

Martell J., C. H. García, y otros tres autores, Estructura Factorial De La Escala De Satisfacción Con La Vida Y Validez Convergente Con La Escala De Calidad De Vida En Preparatorianos Mexicanos, Revista de Psicología y Ciencias del Comportamiento de la Unidad Académica de Ciencias Jurídicas y Sociales, 9(2), 30-45, (2018).

Omar A., L. Paris, y otros tres autores, Validación Del Inventario De Bienestar Subjetivo Con Muestras De Jóvenes $Y$ Adolescentes Argentinos, Brasileros Y Mexicanos, Suma Psicológica, 16(2), 69-84, (2009).

Paiva B. S. R., M. G. de Camargos, y otros cuatro autores, The Pemberton Happiness Index: Validation of the Universal Portuguese Version in a Large Brazilian Sample, Medicine, 95(38), 1-8, (2016).

Peluso D. L., R. N. Carleton y G. J. Asmundson, Depression symptoms in Canadian psychology graduate students: Do research productivity, funding, and the academic advisory relationship play a role?, doi: 10.1037/a0022624, Canadian Journal of Behavioural Science/Revue canadienne des sciences du comportement, 43(2), 119, (2011).

Portolés A. y J. González, Rendimiento Académico Y Correspondencias Con Indicadores De Salud Física Y Psicológica, Sportis. Scientific Journal of School Sport, Physical Education and Psychomotricity, 1(2), 164-181, (2015).

Pu J., H. Hou y R. Ma, The Mediating Effects of Self-Esteem and Trait Anxiety Mediate on the Impact of Locus of Control on Subjective Well-Being, Current Psychology, 36(1), 167-173, (2017).

Revelle W. y R. E. Zinbarg, Coefficients Alpha, Beta, Omega and the Glb: Comments on Sijtsma, doi: 10.1007/s11336008-9102-z, Psychometrika, 74(1), 145-154, (2009).

Rodríguez M. N. y M. A. Ruiz, Atenuación De La Asimetría Y De La Curtosis De Las Puntuaciones Observadas Mediante Transformaciones De Variables: Incidencia Sobre La Estructura Factorial, Psicológica, 29, 205-227, (2008).

Ryff, C. D. Beautiful Ideas and The Scientific Enterprise: Sources of Intellectual Vitality in Research on Eudaimonic WellBeing. In J. Vittersø, Handbook of Eudaimonic Well-Being, Springer International Publishing, pp 95-108, Tromso, Norway, (2016).

Salsman J. M., J.-S. Lai, y otros cuatro autores, et al., Assessing Psychological Well-Being: Self-Report Instruments for the Nih Toolbox, doi: 10.1007/s11136-013-0452-3, Quality of Life Research, 23(1), 205-215, (2014).

Schumacker R. E. y R. G. Lomax, A Beginner's Guide to Structural Equation Modeling: Third Edition, Taylor \& Francis, (2012).

Sheldon K. M. у T. H. Hoon, The Multiple Determination of Well-Being: Independent Effects of Positive Traits, Needs, Goals, Selves, Social Supports, and Cultural Contexts, En The Exploration of Happiness, por A. Delle, pp 141-160 Springer, (2013).

Tov W. y E. Diener, Subjective Well-Being, En Encyclopedia of Cross-Cultural Psychology, Wiley, New York, (2013).

Vitterso, J., The most important idea in the world: an introduction. In J. Vittersø, Handbook of Eudaimonic Well-Being, Springer International Publishing, pp 1-26, Tromso, Norway, (2016).

Wicker P. y B. Frick, The relationship between intensity and duration of physical activity and subjective well-being, doi: 10.1093/eurpub/ckv131, The European Journal of Public Health, 25(5), 868-872, (2015). 